(1)

CrossMark

\title{
Procalcitonin to guide antibiotic administration in COPD exacerbations: a meta-analysis
}

\author{
Alexander G. Mathioudakis ${ }^{1}$, Victoria Chatzimavridou-Grigoriadou², \\ Alexandru Corlateanu ${ }^{3}$ and Jørgen Vestbo ${ }^{1}$
}

Affiliations: ${ }^{1}$ Division of Infection, Immunity and Respiratory Medicine, University Hospital of South Manchester, University of Manchester, Manchester, UK. ${ }^{2}$ Respiratory Department, General Hospital of Nikaia St Panteleimon, Piraeus, Greece. ${ }^{3}$ Department of Respiratory Medicine, State University of Medicine and Pharmacy "Nicolae Testemitanu”, Chisinau, Moldova.

Correspondence: Jørgen Vestbo, Division of Infection, Immunity and Respiratory Medicine, University of Manchester, Education and Research Centre, University Hospital of South Manchester, Manchester, M23 9LT, UK. E-mail: Jorgen.Vestbodmanchester.ac.uk

@ERSpublications

Current evidence suggests that serum procalcitonin could safely halve antibiotic administration in COPD exacerbations http://ow.ly/c693304JkYB

Cite this article as: Mathioudakis AG, Chatzimavridou-Grigoriadou V, Corlateanu A, et al. Procalcitonin to guide antibiotic administration in COPD exacerbations: a meta-analysis. Eur Respir Rev 2017; $26: 160073$ [https://doi.org/10.1183/16000617.0073-2016].

ABSTRACT Challenges in the differentiation of the aetiology of acute exacerbations of chronic obstructive pulmonary disease (AECOPD) have led to significant overuse of antibiotics. Serum procalcitonin, released in response to bacterial infections, but not viral infections, could possibly identify AECOPD requiring antibiotics. In this meta-analysis we assessed the clinical effectiveness of procalcitoninbased protocols to initiate or discontinue antibiotics in patients presenting with AECOPD.

Based on a prospectively registered protocol, we reviewed the literature and selected randomised or quasi-randomised trials comparing procalcitonin-based protocols to initiate or discontinue antibiotics versus standard care in AECOPD. We followed Cochrane and GRADE (Grading of Recommendations, Assessment, Development and Evaluation) guidance to assess risk of bias, quality of evidence and to perform meta-analyses.

We included eight trials evaluating 1062 patients with AECOPD. Procalcitonin-based protocols decreased antibiotic prescription (relative risk (RR) 0.56, 95\% CI 0.43-0.73) and total antibiotic exposure (mean difference (MD) $-3.83,95 \%$ CI $(-4.32--3.35)$ ), without affecting clinical outcomes such as rate of treatment failure (RR 0.81, 0.62-1.06), length of hospitalisation ( $\mathrm{MD}-0.76,-1.95-0.43$ ), exacerbation recurrence rate (RR $0.96,0.69-1.35$ ) or mortality (RR $0.99,0.58-1.69$ ). However, the quality of the available evidence is low to moderate, because of methodological limitations and small overall study population.

Procalcitonin-based protocols appear to be clinically effective; however, confirmatory trials with rigorous methodology are required.

This article has supplementary material available from err.ersjournals.com

Received: Aug 042016 | Accepted after revision: Sept 262016

Support statement: A.G. Mathioudakis was funded by a Fellowship in Guidelines Methodology by the European Respiratory Society (MTF 2015-01). Funding information for this article has been deposited with the Open Funder Registry.

Conflict of interest: Disclosures can be found alongside this article at err.ersjournals.com

Provenance: Submitted article, peer reviewed.

Copyright OERS 2017. ERR articles are open access and distributed under the terms of the Creative Commons Attribution Non-Commercial Licence 4.0. 


\section{Background}

Chronic obstructive pulmonary disease (COPD), a prevalent chronic debilitating disease, is expected to be the fourth leading cause of death by 2030, according to World Health Organization projections [1]. Acute exacerbations of COPD (AECOPD) determine disease morbidity, mortality, healthcare utilisation and associated costs [2]. Prior studies have described different types of exacerbations, such as those driven by bacteria, viruses or by enhanced eosinophilic inflammation [3]. These findings provided a better understanding of the mechanisms of AECOPD and should have led to a more personalised approach in their pharmacological management, which traditionally comprises the combination of bronchodilators and oral corticosteroids with or without the administration of antibiotics [4]. However, the lack of simple tests to aid the distinction between different types of exacerbations in the acute phase has been a significant hindrance. As a result, both oral steroids and antibiotics are administered lavishly in a large proportion of exacerbations, regardless of their cause [5], posing significant and possibly avoidable risks to individual patients and, in the case of antibiotics, to society as well [6].

Procalcitonin, the prohormone of calcitonin, is released in different tissues in response to bacterial, but not viral, infections or nonspecific inflammation [7]. For this reason, procalcitonin is a promising biomarker, which is currently used in the diagnosis of bacterial infections in different settings [8-11]. Such a biomarker could be very useful in the differentiation between COPD exacerbations caused by bacteria and exacerbations with other aetiologies. Nevertheless, its use in COPD could be more challenging, due to the chronically elevated baseline inflammatory status and the chronic bacterial colonisation of the airways [12], and therefore needs to be assessed carefully. Some randomised controlled trials (RCTs) have been conducted to evaluate procalcitonin-based versus standard protocols to guide the decision to initiate or discontinue the administration of antibiotics in patients presenting with AECOPD [13, 14]. The aim of this study was to identify all available RCTs and yield overall effect estimates of different measures of clinical effectiveness of procalcitonin-based protocols to guide the administration of antibiotics in patients with AECOPD.

\section{Methods}

This systematic review and meta-analysis is based on a protocol which was prospectively registered in the PROSPERO register for systematic reviews (www.crd.york.ac.uk/PROSPERO/display_record.asp?ID=CRD4 2016036938) [15]. The present report follows the PRISMA (Preferred Reporting Items for Systematic Reviews and Meta-Analyses) statement [16].

\section{Eligibility criteria}

Eligible studies comprised randomised or quasi-randomised controlled trials comparing procalcitoninbased versus standard protocols to guide the initiation or discontinuation of antibiotics in patients presenting with AECOPD. In addition, we evaluated trials focusing on patients with lower respiratory tract infections or respiratory tract infections in general, and we included subgroups of patients recruited with $A E C O P D$, provided that they included $\geqslant 30$ patients and we were able to acquire adequate data regarding the baseline characteristics and outcomes of these subgroups. We only accepted procalcitonin-based protocols if they included a recommendation to initiate or continue antibiotics for serum procalcitonin levels above a prespecified cut-point and to discontinue or not to initiate for lower levels. Any other protocol used in clinical practice was acceptable as a comparator, provided that the clinicians were unaware of the participants' procalcitonin levels. A previous clinical diagnosis of COPD was considered adequate; AECOPD was defined as a deterioration of the patients' respiratory symptoms that is beyond the normal day-to-day variations and leads to a change in medication. Only studies of patients seeking medical advice in a primary care facility or hospital emergency, respiratory or internal medicine department were accepted. Studies focusing on hospital-acquired infections or studies performed in the intensive care unit (ICU) were excluded. In addition, patients with immunodeficiencies or receiving immune-suppressants (apart from corticosteroids administered for the management of COPD), patients with chronic infections requiring chronic antibiotic therapy and patients with medullary thyroid carcinoma were excluded.

\section{Outcome measures}

The primary outcomes of this meta-analysis included 1) treatment failure for the index exacerbation, defined as deterioration of symptoms, non-improvement or death (within 1-4 weeks) and 2) length of hospitalisation for the index exacerbation. The secondary outcomes included 1) antibiotic exposure for the index exacerbation (including the proportion of patients who were prescribed antibiotics and the duration of the course of antibiotics) and at longest follow-up; 2) antibiotic prescription after an opposite initial decision; 3) re-exacerbation rate at longest follow-up; 4) re-admission rate at longest follow-up; and 5) mortality at longest follow-up. 
The aim of this meta-analysis was to examine whether procalcitonin-guided protocols are associated with a significant decrease in the antibiotic exposure (secondary outcome 1), without significantly affecting any of the clinically important outcomes of an exacerbation (remaining outcomes).

\section{Search strategy and study selection}

We systematically reviewed the electronic databases of Medline, Embase and the Cochrane Central Register of Controlled Trials, using appropriate controlled vocabulary and free search terms to identify studies assessing procalcitonin in patients with COPD (detailed search strategy is described in online supplementary appendix 1). In addition, the World Health Organization International Clinical Trials Registry Platform (ICTRP) search portal, as well as the online registries of abstract proceedings of the European Respiratory Society and the American Thoracic Society and the reference lists of all included trials and all previous relevant systematic reviews that we identified were screened for additional ongoing or completed trials. All databases and online registries were searched from inception to July 19, 2016. Two authors independently screened the titles, abstracts and full text (when appropriate) of all identified articles. Disagreement was resolved through discussion or adjudication by a third investigator when necessary.

\section{Data abstraction}

Two authors independently extracted the following information from each eligible article: full reference and study identifiers, study design, eligibility criteria, predefined outcomes, number and characteristics of the participants and details on the outcomes of interest. Missing data were requested from the authors of the primary studies via e-mail.

\section{Risk of bias and quality of evidence}

Risk of bias of each included study was assessed by two authors independently and discrepancies were resolved through discussion, according to Cochrane guidance. A domain-based evaluation was used, which included the following domains: sequence generation, allocation concealment, blinding of participants and personnel, blinding of outcome assessment, attrition, selective reporting bias and other sources of bias. Explicit judgments were made about the overall risk of bias according to the Cochrane guidance [17]. Risk of bias was assessed separately for different outcomes of interest when that was considered appropriate by the investigators. We were not able to assess the risk of publication bias, due to the small number of included trials $(<10)$.

Quality of evidence for each outcome was assessed using the GRADE (Grading of Recommendations Assessment, Development and Evaluation) methodology, which incorporates risk of bias as well as imprecision, inconsistency, indirectness and publication bias of the body of evidence, using the outcomes as units of analysis [18]. All decisions to downgrade or upgrade the quality of evidence were justified and presented in evidence profile and summary of evidence tables, in accordance with the GRADE guidance. GRADEPro Software (2014; www.gradepro.org) was used in the development of these tables.

According to GRADE working group evaluation, "high quality" evidence suggests that further research is very unlikely to change the confidence in the estimate of effect; "moderate quality" that further research is likely to have an important impact on the confidence in the estimate effect and may change the estimate; "low quality" evidence suggests that further research is very likely to have an important impact on the confidence in the estimate effect and it is likely to change the estimate; and "very low quality" evidence that we are very uncertain of the estimate.

\section{Heterogeneity}

$\mathrm{I}^{2}$ statistic was used to assess statistical heterogeneity within the trials included in each analysis. Substantial heterogeneity $\left(\mathrm{I}^{2}>50 \%\right.$ ) was explored by prespecified subgroup analyses (see later).

\section{Data synthesis}

Data were pooled using the fixed or random effect models in cases where heterogeneity, assessed using the $\mathrm{I}^{2}$ statistic, was found to be $<50 \%$ or $50-75 \%$, respectively. Meta-analyses were not performed in cases of significant unresolved heterogeneity $\left(\mathrm{I}^{2}>75 \%\right)$. Results are presented in the form of relative risk (RR) (95\% $\mathrm{CI}$ ) for dichotomous data and in the form of mean differences (MD) (95\% CI) for continuous data. Meta-analyses were performed using Review Manager 5 (RevMan; http://community.cochrane.org/tools/ review-production-tools/revman-5) software.

\section{Sensitivity and subgroup analyses}

In different prespecified sensitivity and subgroup analyses for all outcomes we included 1) only studies with low risk of bias; 2) only studies with high adherence to the procalcitonin-guided protocols; 3 ) only studies using procalcitonin to guide the initiation of antibiotics; and 4) only studies using procalcitonin to guide the discontinuation of antibiotics. Furthermore, for outcomes measured at longest follow-up, we grouped studies 
with duration of $\leqslant 3$ months versus $>3$ months. Finally, in an additional sensitivity analysis, we repeated the meta-analyses performed using the fixed effects model using the random effects model and vice versa.

\section{Results}

Details of the search results and study selection process are provided in a PRISMA flowchart (online supplementary appendix 2).

\section{Characteristics of the included trials}

Characteristics of the included trials are presented in online supplementary appendix 3. Briefly, eight completed trials evaluating 1062 patients with AECOPD met the inclusion criteria. All were conducted in a hospital setting and recruited patients who had an emergency visit or admission. Five recruited only patients attending with an AECOPD [13, 14, 19-21], while the rest recruited patients with lower respiratory tract infections in general, but included well-characterised and presented subsets of patients who were recruited with an AECOPD [22-24]. Four trials had a follow-up duration of 6 months [13, 14, 20, 22], one only followed the patients during the period of their hospital admission [23] and the rest between 4 and 6 weeks. No significant differences in the baseline characteristics of the patients included in each study arm were reported.

The procalcitonin-based protocols used in all included trials were similar. Antibiotics were recommended for procalcitonin levels $>0.25 \mu \mathrm{g} \cdot \mathrm{L}^{-1}$ and discouraged for levels $<0.25 \mu \mathrm{g} \cdot \mathrm{L}^{-1}$ in seven out of eight studies (NANGIA and GANDHI [21] used a different cut-point, as they recommended antibiotics for procalcitonin levels $\left.>0.5 \mu \mathrm{g} \cdot \mathrm{L}^{-1}\right)$. In addition, in some of the studies antibiotics were strongly discouraged for levels $<0.1 \mu \mathrm{g} \cdot \mathrm{L}^{-1}$ and/or strongly recommended for levels $>0.5 \mu \mathrm{g} \cdot \mathrm{L}^{-1}$. All studies were open labelled or single blinded and the final decision to administer or withhold antibiotics was left to the responsible clinician, who could deviate from the protocol. In three of the included trials procalcitonin levels were used to guide the initiation of antibiotic treatment $[13,21,22]$; in two of the trials, procalcitonin levels guided early discontinuation of antibiotics [14, 23]; and in three trials, procalcitonin levels guided both initiation and discontinuation $[19,20,24]$. Adherence to the procalcitonin-based protocols was reported in four trials and it varied significantly, between $61.3 \%$ and $98.1 \%[19,20,23,24]$.

The strategies used in the control group were similar in all trials but one. VERDURI et al. [14] administered a 10-day course of antibiotics to all participants who were randomised to the control group, while in all other studies the responsible physician, who was unaware of the procalcitonin levels of the participants, had to decide on the administration (or not) of antibiotics, based on the severity of the presentation, clinical signs and symptoms and biochemical and radiological results.

\section{Risk of bias assessment}

Detailed assessment of the risk of bias is depicted in figure 1 and online supplementary appendices 3 and 4. In brief, overall risk of bias was considered high for all included studies, mainly due to performance and detection bias, as none of the included studies was double blinded and the outcomes assessment was only blinded in two studies $[13,24]$. Furthermore, three of the included trials were quasi-randomised, and consequently at high risk of selection bias $[19,20,22]$. Finally, target study population was not reached in one of the trials due to slow recruitment rate [14], while power studies were not reported in two others $[20,21]$. Publication bias was not assessed, because of the relatively small number of studies that contributed to each of the preselected outcomes.

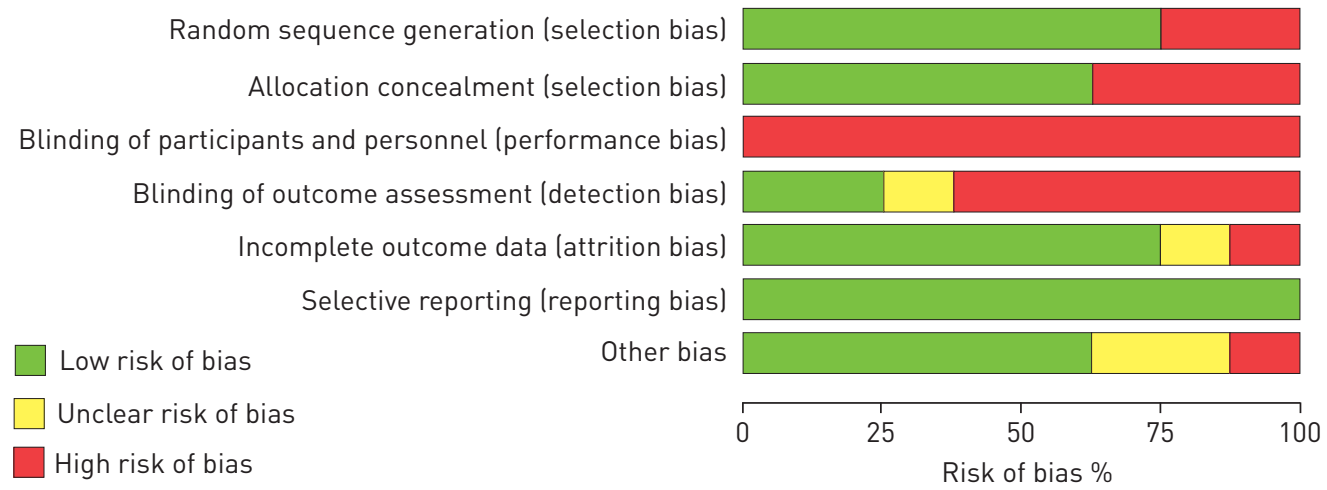

FIGURE 1 Risk of bias graph: authors' judgements about each risk of bias domain, presented as percentages across all included studies. Attrition bias is low for all short-term outcomes of all included trials. 
Data synthesis and quality assessment

Overall effect estimates for each outcome are presented in figure 2 and online supplementary appendix 5. Detailed assessment of the quality of evidence is included in the evidence profile table in online supplementary appendix 6 . A summary of findings is presented in table 1 .

Five studies assessed the rate of treatment failure for the index exacerbation, which was defined as symptom deterioration, non-improvement, admission to ICU or death within 1-4 weeks of recruitment $[13,14,19$, 21, 24]. Treatment failure was observed in 163 out of 834 included patients and no difference was found between the treatment groups ( $\mathrm{RR} 0.81,0.62-1.06 ; \mathrm{I}^{2}=0 \%$, low quality). Only one study, based on a very small number of observations, assessed the proportion of patients who required antibiotic prescription after an initial opposite decision and it did not detect any between-group difference (RR 0.92, 0.21-4.12) [13].

The length of hospital stay for the index exacerbation was reported in all included trials (1062 participants). The mean length varied between 4.5 and 16.06 days in the procalcitonin-guided groups and between 5 and 19.41 days in the control groups. Overall, no significant between-group difference was observed ( $\mathrm{MD}-0.76,-1.95-0.43 ; \mathrm{I}^{2}=59 \%$, moderate quality).

Antibiotic exposure for the index exacerbation was assessed by the proportion of patients who were prescribed antibiotics on admission (or patients who did not discontinue early) and the mean duration of the antibiotic courses. The proportion of patients who received full courses of antibiotics, including patients who were prescribed antibiotics on admission or patients who were not discontinued early, in accordance with each study protocol, was reported in seven of the included trials [13, 14, 19, 21-24], which evaluated 984 patients in total and suggested a $44 \%$ decrease in the antibiotic prescription rate with procalcitonin-guided protocols (RR $0.56,0.43-0.73 ; \mathrm{I}^{2}=84 \%$, moderate quality). Heterogeneity is resolved by excluding the studies by NANGIA and GANDHI [21] and Verduri et al. [14], in which antibiotics were administered to all participants in the control group. The exclusion of these studies had no impact on the overall estimate.

The length of antibiotic exposure for the index exacerbation in days was assessed by six studies [14, 19, 21-24], with an overall study population of 776 participants. A significant decrease of $\sim 4$ days in length of antibiotic exposure was demonstrated in the intervention group compared to standard care (MD $-3.83,-4.32--3.35$; $\mathrm{I}^{2}=58 \%$, moderate quality). This corresponds to a $43 \%$ decrease in antibiotic exposure, given that the mean length of antibiotic exposure in standard care groups was 6.68 days. Two trials assessed the total antibiotic exposure at longest follow-up. STOLz et al. [13] found a reduction in the proportion of patients who received antibiotics during 6 months of follow-up (RR 0.76, 0.64-0.92), while NANGIA and GANDHI [21] found a decrease in the overall length of antibiotic exposure during 6 weeks of follow-up (MD -4.92, -5.94--3.90).

The re-exacerbation and re-hospitalisation rates at the longest follow-up did not differ significantly between the treatment arms. Three studies, including 496 patients, presented the proportion of the participants who had at least one exacerbation during follow-up (RR $0.96,0.69-1.35 ; \mathrm{I}^{2}=0 \%$, low quality) $[14,20,24]$; no between-group difference was found. Three trials ( $\mathrm{n}=398$ participants) assessed the re-hospitalisation rate at longest-follow-up and suggested a trend towards increased rate in the intervention group (RR 1.45, 0.92-2.29; $\mathrm{I}^{2}=0 \%$, low quality) $[14,19,21]$. However, two of these trials also assessed the rate of re-hospitalisation due to an exacerbation at longest follow-up and this was similar between groups (RR 1.22, 0.71-2.09; $\mathrm{I}^{2}=0 \%$, low quality) $[14,19]$.

Finally, mortality at longest follow-up was presented in all included trials (1062 participants). No significant difference was found between study arms (RR 0.99, 0.58-1.69; $\mathrm{I}^{2}=0 \%$, moderate quality).

\section{Sensitivity analyses}

All studies included were deemed to be at high risk of bias, and as such we were not able to perform sensitivity analyses using studies with low risk of bias. However, the trial by LiU and ZHANG [20] was found to be at a significantly higher risk of bias compared to the other included trials (high risk of bias in five out of seven domains assessed and questionable risk of bias for one additional domain). Thus, in an additional analysis, we excluded data from Liu and ZHANG [20]; this did not affect any of the results.

Our results proved robust to each of the remaining predefined sensitivity analyses. Finally, we repeated all analyses excluding data from the trial by NANGIA and GANDHI [21], who used a different cut-point value of procalcitonin $\left(0.5 \mu \mathrm{g} \cdot \mathrm{L}^{-1}\right)$ compared to the remaining trials, but this did not affect any of the results.

\section{Discussion}

The use of procalcitonin-based protocols to guide the decision to administer antibiotics has been evaluated previously in infections of different origins and in different settings [8-10]. A well-conducted systematic review supported the effectiveness of procalcitonin-guided protocols in lower respiratory tract infections, based on evidence of moderate quality according to GRADE assessment [11]. However, these results do not 


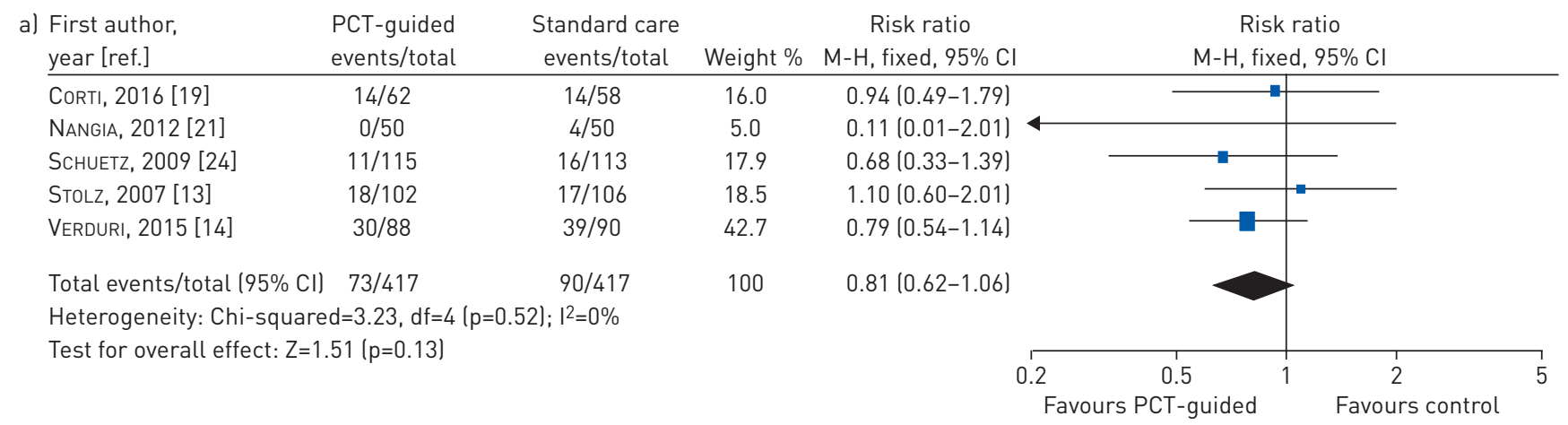

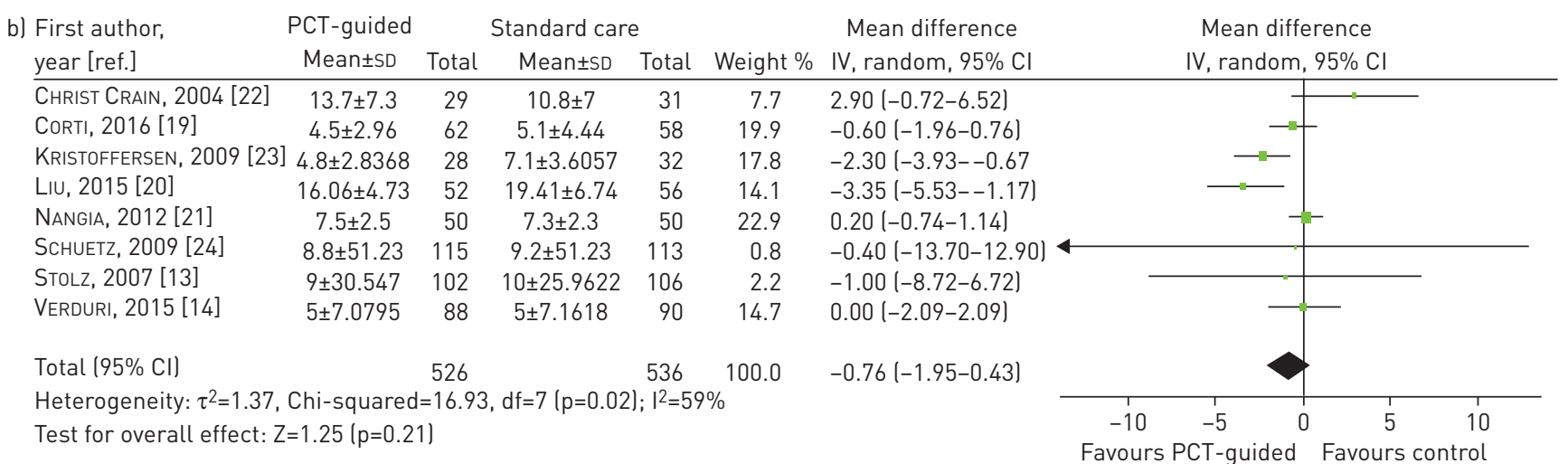

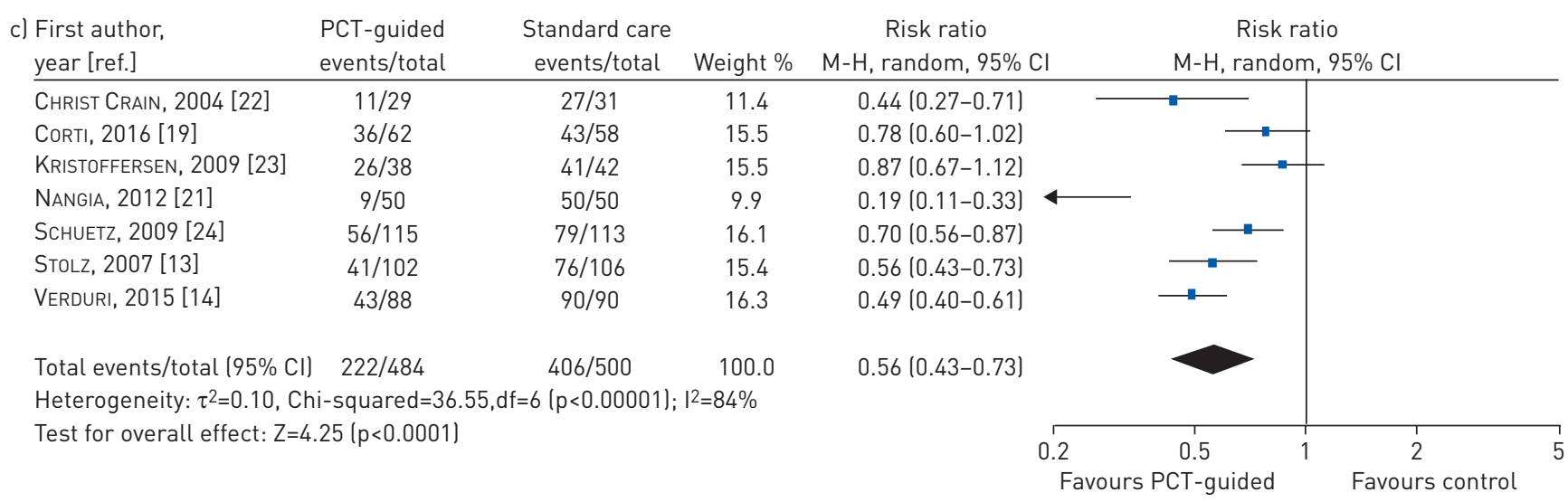

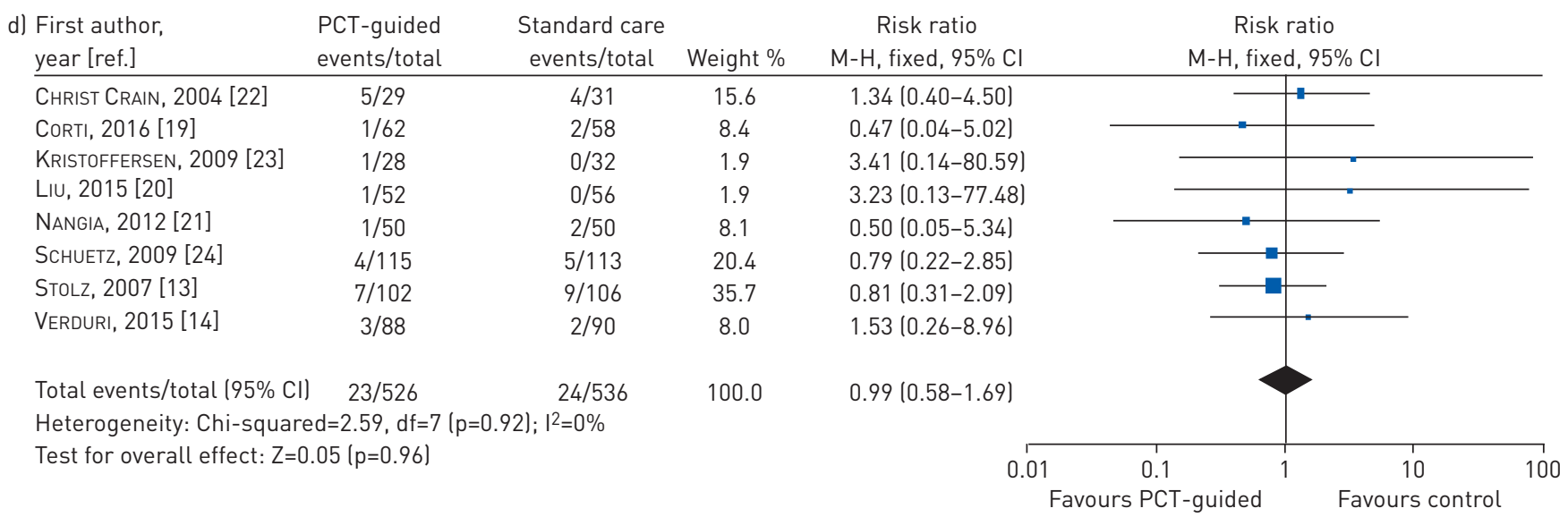

FIGURE 2 Forest plots depicting the overall effect estimates for each outcome. a) Treatment failure for the index exacerbation, defined as symptoms deterioration, non-improvement, intensive care unit admission or death within 1-4 weeks of recruitment; b) length of hospital stay for the index exacerbation; c) antibiotic exposure for the index exacerbation: proportion of patients who were prescribed antibiotics on admission; d) overall mortality at longest follow-up. Data are presented as $\mathrm{n} / \mathrm{N}$, unless otherwise stated. PCT: procalcitonin; $\mathrm{M}-\mathrm{H}$ : Mantel-Haenszel method; df: degrees of freedom. 
TABLE 1 Clinical effectiveness of procalcitonin-based protocols to initiate or discontinue antibiotics in patients presenting with acute exacerbations of chronic obstructive pulmonary disease

$\begin{array}{cccc}\begin{array}{c}\text { Participants } \\ \text { followed-up (studies) }\end{array} & \begin{array}{c}\text { Quality of the } \\ \text { evidence (GRADE) }\end{array} & \begin{array}{c}\text { Relative effect } \\ (95 \% \mathrm{CI})\end{array} & \text { Anticipated absolute effects }\end{array}$

\begin{tabular}{cc}
\multicolumn{2}{c}{ Anticipated absolute effects } \\
\hline $\begin{array}{c}\text { Risk with } \\
\text { standard care }\end{array}$ & $\begin{array}{c}\text { Risk difference with } \\
\text { procalcitonin-guided protocols }\end{array}$
\end{tabular}

\begin{tabular}{|c|c|c|c|c|c|}
\hline $\begin{array}{l}\text { Treatment failure for } \\
\text { the index exacerbation }\end{array}$ & 834 (5 RCTs) & Low $^{\pi,+}$ & RR $0.81(0.62-1.06)$ & 206 per 1000 & $\begin{array}{l}39 \text { fewer failures per } 1000 \\
\text { (78 fewer to } 12 \text { more) }\end{array}$ \\
\hline $\begin{array}{l}\text { Length of hospital stay for } \\
\text { the index exacerbation }\end{array}$ & 1062 (8 RCTs) & Moderate & MD $-0.76(-1.95-0.43)$ & $\begin{array}{l}\text { Mean length of hospital } \\
\text { stay was } 8.55 \text { days }\end{array}$ & $\begin{array}{l}\text { MD } 0.76 \text { fewer days } \\
\text { (1.95 fewer to } 0.43 \text { more) }\end{array}$ \\
\hline $\begin{array}{l}\text { Proportion of patients who were } \\
\text { prescribed antibiotics on admission }\end{array}$ & 984 (7 RCTs) & Moderate & RR $0.56(0.43-0.73)$ & 791 per 1000 & $\begin{array}{l}348 \text { fewer prescriptions per } 1000 \\
\text { (451 fewer to } 214 \text { fewer) }\end{array}$ \\
\hline Duration of the course of antibiotics & 776 (6 RCTs) & Moderate $^{+}$ & MD -3.83 (-4.32--3.35) & $\begin{array}{l}\text { Mean duration of course } \\
\text { of antibiotics was } 8.27 \text { days }\end{array}$ & $\begin{array}{c}\text { MD } 3.83 \text { fewer days } \\
\text { (4.32 fewer to } 3.35 \text { fewer) }\end{array}$ \\
\hline $\begin{array}{l}\text { Exacerbation recurrence rate at } \\
\text { longest follow-up }\end{array}$ & 496 (3 RCTs) & Low ${ }^{\text {1,+ }}$ & RR 0.96 (0.69-1.35) & 205 per 1000 & $\begin{array}{l}8 \text { fewer recurrences } \\
\text { per } 1000 \text { (63 fewer to } 72 \text { more) }\end{array}$ \\
\hline $\begin{array}{l}\text { Re-hospitalisation rate at longest } \\
\text { follow-up }\end{array}$ & 398 (3 RCTs) & Low $^{\text {1,+ }}$ & RR 1.45 (0.92-2.29) & 116 per 1000 & $\begin{array}{l}52 \text { more admissions } \\
\text { per } 1000 \text { ( } 9 \text { fewer to } 150 \text { more) }\end{array}$ \\
\hline $\begin{array}{l}\text { Rate of re-hospitalisation due to an } \\
\text { exacerbation at longest follow-up }\end{array}$ & 298 (2 RCTs) & Low $^{\text {1,+ }}$ & RR 1.22 (0.71-2.09) & 135 per 1000 & $\begin{array}{l}30 \text { more admissions } \\
\text { per } 1000 \text { (39 fewer to } 147 \text { more) }\end{array}$ \\
\hline Overall mortality at longest follow-up & 1062 (8 RCTs) & Moderate $^{+}$ & RR $0.99(0.58-1.69)$ & 41 per 1000 & $\begin{array}{l}0 \text { fewer deaths per } 1000 \\
\text { (18 fewer to } 29 \text { more) }\end{array}$ \\
\hline
\end{tabular}

Data are presented as $n$, unless otherwise stated. GRADE (Grading of Recommendations Assessment, Development and Evaluation) working group quality of evidence assessments are as follows. High quality: further research is very unlikely to change the confidence in the estimate of effect; moderate quality: further research is likely to have an important impact on the confidence in the estimate effect and may change the estimate; low quality: further research is very likely to have an important impact on the confidence in the estimate effect and it is likely to change the estimate; very low quality: the estimate effect is very uncertain. RCT: randomised controlled trial; RR: risk ratio; MD: mean difference. \#: The risk in the intervention group (and its $95 \% \mathrm{CI}$ ) is based on the assumed risk in the comparison group and the relative effect of the intervention (and its $95 \% \mathrm{Cl}$ ); ${ }^{\text {ๆ }}$ : none of the included trials was blinded; ${ }^{+}$: did not meet optimal information size criterion. 
necessary imply that such protocols would also be effective in patients presenting with AECOPD, since the chronically elevated baseline inflammatory status and the chronic bacterial colonisation of the airways of patients with COPD [12] could possibly affect the serum procalcitonin levels. We conducted a systematic review and meta-analysis to evaluate the clinical effectiveness of procalcitonin-based protocols in patients presenting with AECOPD. Our findings suggest that procalcitonin-based protocols may be superior to standard care in these patients. More specifically, we showed that procalcitonin guidance significantly limits and targets the antibiotic exposure, without any obvious impact on the clinical outcomes (such as treatment success, re-exacerbation, re-hospitalisation or mortality). However, the quality of available data is low to moderate, suggesting that additional appropriately powered confirmatory trials with rigorous methodology are required before recommending introducing such strategies in daily clinical practice.

Such an intervention could make a significant difference in the management of patients with AECOPD. Currently, owing to the challenges of distinguishing bacterially induced AECOPD from AECOPD with other aetiology in the acute phase, both antibiotics and oral corticosteroids are administered to most patients presenting with what is regarded a moderate or severe AECOPD. Nevertheless, the aetiology of AECOPD varies and bacterial infections, where antibiotics are indicated, appear to account for approximately half of them [3]. This suggests that antibiotics are significantly overused in AECOPD, posing significant risks to individual patients and society [6]. Importantly, COPD patients are more prone to the risks associated with overuse of antibiotics, because of chronic airway colonisation with bacterial strains which tend to progressively become less sensitive to antibiotics. In addition, some of these patients may be more prone to infections, due to defects in their immune system [25], and this will expose them to frequent administration of antibiotics in any case.

The majority of patients with moderate or severe AECOPD also receive courses of oral corticosteroids. Previous studies have shown that oral corticosteroids confer mild or moderate advantage over placebo when administered in unselected patients with AECOPD [26, 27]. However, they are also associated with numerous well-known adverse events, such as the risk of pneumonia. It has been hypothesised that oral steroids may be efficacious in patients with inflammatory, non-infective exacerbations, and not in AECOPD induced by bacteria [28]. However, this has not yet been evaluated in an RCT, probably due to the challenges of the identification of the type of exacerbation in the acute phase. All in all, it appears that procalcitonin-based protocols could contribute to the personalisation of the management of COPD exacerbations, decrease the use of both antibiotics and oral corticosteroids and may enable the design and conduct of new clinical studies that will provide new insights into the mechanisms and management of AECOPD.

A number of previous studies failed to demonstrate clinically significant benefits of the administration of antibiotics in AECOPD, leading some experts to question their usefulness [29]. However, well-conducted meta-analyses of RCTs suggested small but significant decreases in treatment failure and mortality rates and time to recovery [30, 31]. Moreover, all these trials and meta-analyses included unselected exacerbations, and only half of them are anticipated to have been induced by bacteria and responsive to antibiotics. This suggests that the real magnitude of effects in bacterially induced exacerbations has been significantly underestimated.

Others proposed that a sensible and strict antibiotic stewardship policy alone might effectively reduce antibiotic administration in patients presenting with AECOPD. However, previous extensive campaigns against inappropriate antibiotic prescribing in respiratory tract infections led to only modest decrease in the prescription rates [32, 33]. In addition, a subanalysis of the extensive European COPD Audit [5] reported that $61.4 \%$ of the included (hospitalised) AECOPD were fulfilling Global Initiative for Chronic Obstructive Lung Disease criteria for antibiotic administration. In our meta-analysis, only $45.6 \%$ of the participants who were hospitalised for AECOPD received antibiotics following procalcitonin-guided protocols; this suggests procalcitonin guidance might confer an additional decrease in antibiotic prescription $(\leqslant 15 \%)$, compared to effective stewardship.

Recent cross-sectional studies noted significant discrepancies between procalcitonin levels and sputum culture results and raised concerns regarding the effectiveness of procalcitonin protocols to guide antibiotic administration in patients with COPD [34, 35]. However, this study design is not appropriate to assess procalcitonin protocols, and, more importantly, the sensitivity and specificity of sputum cultures to recognise AECOPD induced by bacteria is far from ideal. First, the airways of COPD patients are frequently colonised with bacteria, which may result in false positive results [36]. Second, the sensitivity of sputum cultures is limited in patients with COPD [37].

An additional relevant RCT did support our findings, although it did not meet the eligibility criteria. WANG et al. [38], in a blinded RCT involving 194 patients presenting with AECOPD and procalcitonin levels $<0.1 \mu \mathrm{g} \cdot \mathrm{L}^{-1}$, compared antibiotics versus control and found similar success rates and similar safety outcomes 30 days after discharge. These results oppose the use of antibiotics in patients with AECOPD and low procalcitonin levels. 
A meta-analysis assessed the cost-effectiveness of the use of serum procalcitonin to guide antibiotic therapy in other infections and estimated the cost of procalcitonin to be $\sim £ 11(€ 14)$ per measurement in the United Kingdom [26]. We consider this an acceptable cost, given the expected net benefits in the management of patients with an AECOPD.

We recognise that our meta-analysis has some limitations. First, a high risk of bias is introduced by the fact that none of the included trials were double blinded. The reasons probably include challenges in the design of a double-blinded trial to assess a biomarker-guided protocol (complexity in the allocation of appropriate treatment for each patient, challenges in blinding clinicians to laboratory results, etc.), significant associated costs and, for the earlier studies, lack of adequate safety data to support a double-blinded design (clinicians could deviate from the protocol in all included studies). Second, our results are imprecise, due to the low overall study population and event rates. Imprecision was judged using the optimal information size criterion, the study population generated by conventional power calculation for a single, adequately powered trial, in accordance with the GRADE guidance [18]. For all clinically important outcomes of exacerbations, we used narrow non-inferiority margins (2\% for mortality and $5 \%$ for other outcomes) in the calculation of the optimal information size. Most trials use less strict criteria (i.e. $5 \%$ and 10\%, respectively) and such criteria would have not resulted in downgrading the quality of evidence for imprecision. However, we consider strict margins more appropriate in the setting of a meta-analysis and we wanted to account for the relatively low adherence to the procalcitonin-based protocols that was observed in some of the included trials. Consequently, our results are of low-to-moderate quality, according to GRADE, suggesting that additional, well-designed and conducted trials are required to confirm our findings.

However, all outcomes support the intervention and all included studies presented consistent results. These observations can reassure that procalcitonin protocols are very unlikely to be associated with significant risks and highlight the need for further more rigorous research with the aim of introducing this intervention to clinical practice in the near future.

\section{Conclusion}

Our findings suggest that procalcitonin-based protocols to guide the initiation (or discontinuation) of antibiotics in patients presenting with acute exacerbations of COPD appear to be clinically effective and safe. The quality of the available evidence is low to moderate, because of the methodological limitations and small study populations of the available trials. Given the potential impact on the management of these patients, the need for additional appropriately designed and powered confirmatory randomised controlled trials cannot be stressed enough.

\section{Acknowledgements}

The investigators would like to cordially thank Kristina Kristoffersen (Aarhus University Hospital, Aarhus, Denmark), Vivek Nangia (Fortis Hospital, Vasant Kunj, New Delhi, India), Philipp Schuetz (Medical Faculty of the University of Basel, Basel, Switzerland) and Daiana Stolz (University Hospital Basel) for providing additional data from their studies.

\section{References}

1 Mathers CD, Loncar D. Projections of global mortality and burden of disease from 2002 to 2030. PLoS Med 2006; 3: e442.

2 Wedzicha JA, Wilkinson T. Impact of chronic obstructive pulmonary disease exacerbations on patients and payers. Proc Am Thorac Soc 2006; 3: 218-221.

3 Bafadhel M, McKenna S, Terry S, et al. Acute exacerbations of chronic obstructive pulmonary disease: identification of biologic clusters and their biomarkers. Am J Respir Crit Care Med 2011; 184: 662-671.

4 Vestbo J, Hurd SS, Agustí AG, et al. Global strategy for the diagnosis, management, and prevention of chronic obstructive pulmonary disease: GOLD executive summary. Am J Respir Crit Care Med 2013; 187: 347-365.

5 López-Campos JL, Hartl S, Pozo-Rodriguez F, et al. Antibiotic prescription for COPD exacerbations admitted to hospital: European COPD audit. PloS One 2015; 10: e0124374.

6 Llor C, Bjerrum L. Antimicrobial resistance: risk associated with antibiotic overuse and initiatives to reduce the problem. Ther Adv Drug Safety 2014; 5: 229-241.

7 Biju PG, Garg S, Wang W, et al. Procalcitonin as a predictive biomarker for total body irradiation-induced bacterial load and lethality in mice. Shock 2012; 38: 170-176.

8 van der Does Y, Rood PP, Haagsma JA, et al. Procalcitonin-guided therapy for the initiation of antibiotics in the ED: a systematic review. Am J Emerg Med 2016; 34: 1286-1293.

9 de Jong E, van Oers JA, Beishuizen A, et al. Efficacy and safety of procalcitonin guidance in reducing the duration of antibiotic treatment in critically ill patients: a randomised, controlled, open-label trial. Lancet Infect Dis 2016; 16: 819-827.

10 Schuetz P, Müller B, Christ-Crain M, et al. Procalcitonin to initiate or discontinue antibiotics in acute respiratory tract infections. Evid Based Child Health 2013; 8: 1297-1371.

11 Schuetz P, Muller B, Christ-Crain M, et al. Procalcitonin to initiate or discontinue antibiotics in acute respiratory tract infections. Cochrane Database Syst Rev 2012; 9: CD007498.

12 Desai $\mathrm{H}$, Eschberger $\mathrm{K}$, Wrona $\mathrm{C}$, et al. Bacterial colonization increases daily symptoms in patients with chronic obstructive pulmonary disease. Ann Am Thorac Soc 2014; 11: 303-309. 
13 Stolz D, Christ-Crain M, Bingisser R, et al. Antibiotic treatment of exacerbations of COPD: a randomized, controlled trial comparing procalcitonin-guidance with standard therapy. Chest 2007; 131: 9-19.

14 Verduri A, Luppi F, D'Amico R, et al. Antibiotic treatment of severe exacerbations of chronic obstructive pulmonary disease with procalcitonin: a randomized noninferiority trial. PloS One 2015; 10: e0118241.

15 Mathioudakis AG, Chatzimavridou-Grigoriadou V, Corlateanu A, et al. Serum procalcitonin to guide antibiotic treatment in acute exacerbations of chronic obstructive pulmonary disease. PROSPERO 2016: CRD42016036938.

16 Liberati A, Altman DG, Tetzlaff $\mathrm{J}$, et al. The PRISMA statement for reporting systematic reviews and meta-analyses of studies that evaluate health care interventions: explanation and elaboration. Ann Intern Med 2009; 151: W65-W94.

17 Higgins JPT, Altman DG, Sterne JAC. Assessing risk of bias in included studies. In: Higgins JPT, Green S, eds. Cochrane Handbook for Systematic Reviews of Interventions version 5.1.0. London, The Cochrane Collaboration, 2011.

18 Balshem H, Helfand M, Schünemann HJ, et al. GRADE guidelines: 3. Rating the quality of evidence. J Clin Epidemiol 2011; 64: 401-406.

19 Corti C, Fally M, Fabricius-Bjerre A, et al. Point-of-care procalcitonin test to reduce antibiotic exposure in patients hospitalized with acute exacerbation of COPD. Int J Chron Obstruct Pulmon Dis 2016; 11: 1381-1389.

20 Liu SS, Zhang YB. The value of serum procalcitonin level in guiding the use of antibiotic in patients with acute exacerbation of chronic obstructive pulmonary disease. Chinese J Antibiot 2015; 40: 459-463.

21 Nangia V, Gandhi K. Use of procalcitonin to guide the antibiotic therapy in patients with an acute exacerbation of COPD in a resource-limited setting: a case-control study. Clin Microbiol Infect 2012; 18: Suppl. 3 O404.

22 Christ-Crain M, Jaccard-Stolz D, Bingisser R, et al. Effect of procalcitonin-guided treatment on antibiotic use and outcome in lower respiratory tract infections: cluster-randomised, single-blinded intervention trial. Lancet 2004; 363: 600-607.

23 Kristoffersen KB, Søgaard OS, Wejse C, et al. Antibiotic treatment interruption of suspected lower respiratory tract infections based on a single procalcitonin measurement at hospital admission - a randomized trial. Clin Microbiol Infect 2009; 15: 481-487.

24 Schuetz P, Christ-Crain M, Thomann R, et al. Effect of procalcitonin-based guidelines $v s$ standard guidelines on antibiotic use in lower respiratory tract infections: the ProHOSP randomized controlled trial. JAMA 2009; 302: 1059-1066.

25 Fan VS, Gharib SA, Martin TR, et al. COPD disease severity and innate immune response to pathogen-associated molecular patterns. Int J Chron Obstruct Pulmon Dis 2016; 11: 467-477.

26 Aaron SD, Vandemheen KL, Hebert P, et al. Outpatient oral prednisone after emergency treatment of chronic obstructive pulmonary disease. N Engl J Med 2003; 348: 2618-2625.

27 Niewoehner DE, Erbland ML, Deupree RH, et al. Effect of systemic glucocorticoids on exacerbations of chronic obstructive pulmonary disease. N Engl J Med 1999; 340: 1941-1947.

28 Agusti A, Bel E, Thomas $\mathrm{M}$, et al. Treatable traits: toward precision medicine of chronic airway diseases. Eur Respir J 2016; 47: 410-419.

29 Daniels JM, Snijders D, de Graaff CS, et al. Antibiotics in addition to systemic corticosteroids for acute exacerbations of chronic obstructive pulmonary disease. Am J Respir Crit Care Med 2010; 181: 150-157.

30 Saint S, Bent S, Vittinghoff E, et al. Antibiotics in chronic obstructive pulmonary disease exacerbations. A meta-analysis. JAMA 1995; 273: 957-960.

31 Ram FS, Rodriguez-Roisin R, Granados-Navarrete A, et al. Antibiotics for exacerbations of chronic obstructive pulmonary disease. Cochrane Database Syst Rev 2006; 19: CD004403.

32 Schroeck JL, Ruh CA, Sellick JA Jr, et al. Factors associated with antibiotic misuse in outpatient treatment for upper respiratory tract infections. Antimicrob Agents Chemother 2015; 59: 3848-3852.

33 Wutzke SE, Artist MA, Kehoe LA, et al. Evaluation of a national programme to reduce inappropriate use of antibiotics for upper respiratory tract infections: effects on consumer awareness, beliefs, attitudes and behaviour in Australia. Health Promot Int 2007; 22: 53-64.

34 Falsey AR, Becker KL, Swinburne AJ, et al. Utility of serum procalcitonin values in patients with acute exacerbations of chronic obstructive pulmonary disease: a cautionary note. Int J Chron Obstruct Pulmon Dis 2012; 7: 127-135.

35 Chang $\mathrm{CH}$, Tsao KC, Hu HC, et al. Procalcitonin and C-reactive protein cannot differentiate bacterial or viral infection in COPD exacerbation requiring emergency department visits. Int J Chron Obstruct Pulmon Dis 2015; 10: 767-774.

36 Bafadhel M, Haldar K, Barker B, et al. Airway bacteria measured by quantitative polymerase chain reaction and culture in patients with stable COPD: relationship with neutrophilic airway inflammation, exacerbation frequency, and lung function. Int J Chron Obstruct Pulmon Dis 2015; 10: 1075-1083.

37 Shimizu K, Yoshii Y, Morozumi M, et al. Pathogens in COPD exacerbations identified by comprehensive real-time PCR plus older methods. Int J Chron Obstruct Pulmon Dis 2015; 10: 2009-2016.

38 Wang JX, Zhang SM, Li XH, et al. Acute exacerbations of chronic obstructive pulmonary disease with low serum procalcitonin values do not benefit from antibiotic treatment: a prospective randomized controlled trial. Int $J$ Infect Dis 2016; 48: 40-45. 